\title{
Versions en català de constitucions de Pau i Treva
}

\section{Gener Gonzalvo i Bou}

Axiu Històric Comarcal de Tàrrega.

C/ De les Sitges, 4-6, 25300 Tàrrega. Spain

\section{Resum}

L'article ofereix una nova contribució a l'estudi de les constitucions de Pau i Treva. L'autor mostra dues constitucions escrites en català de la primeria del segle XIII.

Abstract. Catalan's versions of contitutions of Pau i Treva

This paper tries to offer a new contribution in the study of constitutions of Pau $i$ Treva. The author shows two constitutions wrote in Catalan of the early 13 th century.

Ja fa alguns anys que vam veure la necessitat de realizzar una edició crítica de les constitucions de Pau i Treva de Catalunya dels segles XI al XIII, un cop publicat, el 1986, un estudi nostre sobre aquesta institució de la Catalunya medieval ${ }^{1}$. Per encàrrec del Departament de Justícia de la Generalitat vam realitzar aquest treball crític, que es va editar el 1994 a la col-lecció «Textos Jurídics

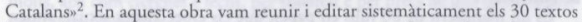
llatins de les constitucions de Pau i Treva conegudes fins avui, en el període en què la institució es va mantenir amb una vigència important (segles $\mathrm{XI}, \mathrm{XII}$ i XIII).

En el decurs de l'edició crítica, ens vam adonar, però, que hi havien algunes qüestions encara pendents, i que calia que en el futur s'anessin resolent. Aixi, l'estudi de la incorporació d'alguns textos de Pau i Treva dins els Usatges de Barcelona, el tema de les reunions de la cúria reial, derivades de les antigues assemblees comtals, i, per tant, l'origen de la Cort General de Catalunya, i, finalment, l'anàlisi de les versions en català dels estatuts de la Pau i Treva.

1. Gonzalvo i Bou, Gener. La Pau i Treva a Catalunya. Origen de les Corts Catalanes. Barcelona: La Magrana/Institut Municipal d'Història, 1986. Aquest treball fou el resum de la nostra tesi de llicenciarura, dirigida pel Dr. Frederic Udina i Martorell.

2. Gonzalvo I Bou, Gener. Les Constitucions de Pau i Treva de Catalunya (segles XI-XIII). Barcelona: Departament de Justicia de la Generalitat, 1994. 
El motiu d'aquest pecit article és el d'aportar dos textos catalans d'aquestes constitucions de Pau i Treva, localitzats durant el nostre treball de recerca per la publicació crítica dels originals llatins dels estatuts. Un d'ells és lassemblea de Cervera de 1202, sota el regnat de Pere I, l'altre correspon a la Cort General de Barcelona de 1228, presidida pel rei Jaume I. Com és obvi, ens estalviem aquí de ressenyar el context històric i el contingut jurídic d'aquestes assemblees ${ }^{3}$. Si que cal, però, comentar el fet que no són massa abundoses les versions catalanes de constitucions jurídiques, almenys amb anterioritat a la seva traducció oficial del segle XV, que recullen les compilacions oficials de les Constitucions de Catalunya. Si no són abundoses, no vol dir que no n'existissin algunes versions. Per exemple, es coneixen algunes constitucions de Pere I i Jaume I en versió catalana del segle XIII ${ }^{4}$. D'altra banda, les traduccions cata-

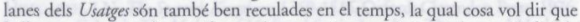
la llengua catalana s'anava fent cada cop més necessària en els usos judicials del nostre pais 5 .

Com dèiem, durant la nostra recerca vam localitzar aquestes versions catalanes de les assemblees de 1202 i 1228 . Això vol dir que, de ben segur, un seguiment encara més profund ens pot permetre més troballes, com per exemple, en manuscrits de la Biblioteca Nacional de París, o en els de la Reial Acadèmia de la Història de Madrid. És tanta la quantitat i qualitat de manuscrits juridics catalans medievals, que forçosament ens inclinem a pensar que encara resta molt camí per fer en la recerca de textos en versió catalana.

Vam localitzar aquests textos catalans en el Manuscrit Z-III-14, de la Biblioteca del monestir d'El Escorial. És un códex ja conegut al segle xIx per Benvingut Oliver ${ }^{6}$. El volum, de fet, és més conegut perquè hi consta una versió catalana dels Usatges de Barcelona, en els seus primers 23 folis, utilitzada primer per encapçalar la col-lecció de les corts catalanes de l'Acadèmia de la Historia de Madrid ${ }^{7}$, i per Josep Rovira i Ermengol en la seva edició dels Usatges de Barcelona ${ }^{8}$.

Es un manuscrit de 80 fulls en paper, que els especialistes actuals situen entre el final del segle XIII i el principi del XIV. Està escrit a plana sencera, amb un format de $227 \times 145 \mathrm{~mm}$, amb capítols i rúbriques en vermell, i amb uti-

3. Podeu consultar el nostre primer treball de 1986, p. $66-67$ (Cervera, 1202) i 82-86 (Barcelona, 1228).

4. GuDiot, Josep. «Traducció dels Usatges, les més antiges Constitucions de Catalunya y les Costumes de Pere Alberts. Dins Anuari de l"Institur d'Estudis Catalans, I (1907), p. 285-317.

5. Sobre els Usatges, vegeu: BASTARDAS, Joan. Usatges de Barcelona. El Codi a mitjan segle XII. Barcelona: F. Noguera, 1984.

6. OUVER I EstreIÉs, Benvingut. Historia del derecho en Cataluena, Mallonca y Valencia. Madrid: 1876.

7. Cortes de los antiguos reinos de Aragón y de Valencia y principado de Cataluña publicadas por la Real Academia de la Historia. Madrid: 1890, vol. I, la part, p. 731-758.

8. Usarges de Barcelona $i$ Commemoracions de Pere Albert. Barcelona: Barcino, 1933, p. 33-34. L'editor ja ens fa notar que el manuscric, per la seva conservació, dóna la impressió d'haverse utilizat molt. També vegeu, Joan BASTARDAS, op. cit., p. 15. 
lització de calderons. Conté, a més dels Usatges, textos de constitucions i corts de Catalunya, i acaba amb la Cort General de Barcelona del 1300. Cal dir a més que també copia la versió llatina de les constitucions de Pau i Treva aprovades a la Cort General de Tarragona del 1235. Com a curiositat, direm que hi ha un dibuix d'un comte de Barcelona (possiblement Ramon Berenguer III), a cavall, amb profusió de l'ús de l'estendard de Sant Jordi'.

Com és lògic, hem consultat les versions catalanes oficials de les diferents compilacions de les Constitucions de Catalunya. En un primer moment, vam voler ressenyar les variants textuals entre el text d'El Escorial i les traduccions oficials; aquestes variants, però, eren tan nombroses, que hem preferit no recollir-les, per no carregar massa els textos, tot deixant aquest punt per a la comprobació dels estudiosos que vulguin aprofundir en el tema ${ }^{10}$. Sense més preàmbuls, doncs, donem la transcripció d'aquestes constitucions, esperant que en el futur se'n puguin localitzar d'altres en llengua catalana que contribueixin a l'avenç dels nostres estudis històrics, juridics i, àdhuc, filologics.

\section{2, setembre. Cervera}

Constitucions aprovades per Pere I en una assemblea de la cúria reial, formada per prelats $i$ magnats, a Cervera. Alguns d'aquests estatuts afecten la Pau $i$ Treva.

A. Versió catalana del final del segle XIII o principi del segle XIV: Biblioteca del monestir d'El Escorial, Ms. Z-III-14, fol. 32 r. -33 . v. ${ }^{11}$

Constitucio Cervarie ${ }^{12}$. Anno Domini millesimo duocentesimo secundo. En lo mes de setembre, en la onrable cort a Cervera, present larchabisbe de Taragona, e de Narbona, G. de Cardona, G. de Cervelyo, Arbert de Castellvii, R. de Cervera, G. Saguardia, Bernat Saportella, G. de Torroya, R. Gauceran, e altres molts, així com clergues e lechs, per volentat e per precs de tots que ainstantment o demanaven.

[I]. Establí lo senyor rei en P., per la gràtia de Déu rey d'Aragó e comte de Barçelona, que no reeba sots la senyoria sua altruy hom o sots la sua imperància, sens voluntat e licència del senyor del dit hom.

9. Vegeu la descripció d'aquest manuscrit a Zarco Cuevas, Julián. Canálogo de los manuscritos catalanes, valencianos, gallegos y portugueses de la Biblioteca de El Escoriah Madrid: 1932, p. 99-102. Vegeu també: FuUViג, Armand de. Els quatre pals. L'escut dels comtes de Barcelona. Barcelona: Dalmau, 1994, p. 70.

10. Cervera, 1202. Constitucions de Catalunya. Incunable de 1495, fol. CCXXVII r.-v. (1a compilació) i Constitucions y altres drets de Cathalumya, Barcelona, 1704 (3a compilació), I, 10, 11, 5. - Barcelona, 1228. Constitucions de Catalunya, Incunable de 1495, fol. CCXXXIII v.CCXXXV v., i Constitucions y altres drets de Cathalunya, I, 10, 11, 7.

11. Els folis 32 v. i $33 \mathrm{r}$, són en blane.

12. Al marge esquerre, hi figuren les armes dels Cervera. 
(II]. Aquí metex, no [corrupiblement ${ }^{13}$ establi que si los senyors los lurs payeses malament tractaran, o les lurs cosses a els tolran, axí aquelles coses qui són en pau e en treva, com les altres, no sien tenguts en alcuna manera al senyor rey, si donchs del feu del senyor rey, o de locs religioses, la doncs los favaters no agesen leer de fer aquella cossa.

[III]. Enadí encara, e fermament manà, que alcú no gosàs metre foch a blat ni en ajustament de blat, qui vulgarmenr és dit modolo o garbera, la qual cossa qui farà sia gitat de pau e de treva del senyor rei, sí e les sues quosas, en tot loch sien destruides los seus lochs. E si los homens del senyor rey o de lochs religioses en penyora los dits loschs tendran, o honors de aquels, sien destruïts los lochs e les fortalees d'aquels de tot en tot. Mas les planees e les honors d'aquels als creedors seus, salves e no corrompudes romanguen.

[IV]. Encara establí que leguda cossa sia a cascú, que si atrobarà bèsties d'arar, o qualsquns placie altres en malafeyta sua, qui és dit vulgarment tala, aquelles pendre puxe, e tant longament tenir d'entró que así sia satisfet per lo senyor de les bèsties.I fol. $33 \nu$.

[V]. En aquesta metexa cort establim que bous àrecs de cavalers fosen sots pau tan solament, les altres cosses emperò no fossen sots pau e treva.

\section{8, desembre, 21. Barcelona}

Constitucions de Pau i Treva promulgades per Jaume I, amb el consell dels prelats, magnats $i$ prohoms de les ciutats $i$ viles de Catalunya, en una cort general celebrada a Barcelona. Les constitucions són vàlides per a tot Catalunya, perpetuament, des del Cinca fins a Salses.

A. Versió catalana del final del segle XIII o principi del segle XIV: Biblioteca del monestir d'El Escorial, Ms. Z-III-14, fol. 34 r. $-38 \mathrm{v}$.

\section{Constitucio ${ }^{14}$ de Barçelona, de pau e de treva}

In Christi nomine. Sia a tots manifest que nos en Iacme, per la gràcia de Déu rey d'Aragó, comte de Barcelona e senyor de Monpesler, volen dels ancessors nostres seguir la via, la manera, els eximplis, ab consel dels honrats pares nostres, n'Espàreg, archebisbe de Terragona, e d'en Berenguer, bisbe de Barçelona, e d'en G., bisbe de Vich, e d'en G., bisbe de Gerona, e de abbats, e de nobles barons, d'en Nuno Sanç, comte ${ }^{15}$ de Roseyó, d'en Hugo, comte d'Ampúries, e d'en G. de Munchada, e d'en G. de Cervera, e d'en Huc de Mataplana, e

13. Paraula de lectura dubtosa, per mala conservació del manuscrit.

14. Al marge esquerre hi figuren les armes de la ciutat de Barcelona.

15. Comte de Roseyó, interlineat. 
d'en G. de Cervelyó, e d'en R. Alamany, e d'en G. de Clarmunt, e de molts altres cavalers e de ciutadans e d'altres promens de viles, de Cincha entró a Salses establiren en aquesta forma.

[I]. En axí que totes les esgleyes e les persones dels clergues de qualque orde sien ab totes les lurs cosses e drets, sots aquesta pau sien establides.

[II]. Encara los siminteris e les sacreres vel sacraria, de qualque esgleya entorne d'aquela establides, negú no asaig esvair ne trencar-ne neguna cosa d'aquen trer [sic]. Als trencadors emperò d'aquest establiment, pena de sacrilegi los sia posada, e per lo bisbe d'aquel bisbat sien destrets de satisfer lo dan en doble a aquel a qui serà lo dan donat.

[III]. Les esgleyes encara encastelades, sots defeniment de pau e de treves establim, en així emperò que si robadors o ladres en aqueles esgleyes preça, o altres malafetes ajustaran, los clams sien fets/ fol. $34 v$. e mostrats al bisbe d'aquel bisbat on fet serà, e a nos al nostre batle e la donchs per nostre juy o d'aquel jutge a qui comanat serà sie esmenat, o si no de pau e treves les esgleyes damunt dites sien gitades.

[IV]. Los domenges encara, dels canonges e dels monestirs, sots seguretat de pau e de treves establim, e dobla pena de restitució apareyen a aquels qui aqueles gosaran esvayr.

[V]. Clerges, monyos, vidues, pubils, orfens, moniesses donades, ab totes les lurs cosses, sots defeniment de pau e treves establits, esgardan que negú aqueles no penrà, ne neguna injúria a els no faça, si dons no seran atrobats en malefici. E si negú en alcú d'aquests, mans forçades gitarà, o alcuna cossa a els tolrà, en doble la tolta restituesca, e de injúria no menys al juy del bisbe del bisbat en que fet serà, a aquels satisfaça. La pena, emperò, de sacriligi, al bisbe sia dada.

[VT]. Les emunitats o franchees del Temple e del Espital de Iherusalem e dels altres lochs honrats, els honrats frares del Temple e del Espital, e dels altres lochs honrats, ab totes les lurs cosses, sots aquel defeniment, e pau e treva, egualment ab clergues o ab esgleyes establim.

[VII]. Homens e fembres de vila, d'esgleyes, o de lochs religioses, e de canonges, e nostres, e totes les lurs cosses, axí mobles com se movens, so és saber, bous, oveyles, asens, someres, cavayls, egues, e totes altres bèsties, sien covinens a arar, o no, sots seguretat de pau e treves en axí establim, que negú aqueles persones/fol. 35 r. no penrà ne esvaescha, ne en cors propi ne en cosses lurs mobles o no mobles, dan 
no do, si donchs no seran atrobats en maleficis o encara que.n iran en cavalcades $a b$ lurs senyors o ab altres. Mas pus seran retornats als lurs alberchs, sots la damunt dita pau romangen.

[VIII]. Ciutadans, emperò, e burgeses, e tots homens nostres, e de les viles nostres, ab homens e ab les cosses d'aquels, mobles e no mobles, jueus encara, $a b$ totes les lurs cosses, pubils, vídues e orphens, ab homens e ab les cosses d'aquels, sots la pau nostra establim.

[IX]. Vilans e vilanes de cavalers, e la lur companya, sots la damunt dita pau establim, si donchs no seran atrobats ab armes.

[X]. Totes bèsties areges, tan solament, e estrumenta o arteri d'arar, colomers, paylers, abeylers, olivers, molins, en la damunt dita pau establim.

[XI]. Encara negú no penyor ne prena bèsties areges ne arreu d'arar, per fermança ne per propi deute, ne per altrui ne per propi malefici ne per malefici ne per deute de lurs senyors, ne encara si especialment seran obligades.

[XII]. Pageses emperò, ne la lur companya, per deutes de lurs senyors, ne per propis ne per fermances, en neguna manera personalment no sien preses, ne preses retenguts.

[XIII]. Encara negú encenna a cases ne en altra manera foch no pos a noure a negune coses. I fol. $35 \mathrm{v}$.

[XIV]. Si emperò, los barons nostres o cavalers, entre els gerra auran, e a combatre castel o forssa de lurs enemichs vendran, e combaten en lo cors del castel o de la força, foch possaran, no sien tenguts per pau trenchada.

[XV]. Terres en contenció possades, negú lo les laure, pas que comonides li seran per aquel en lo qual justicia de plet no romandrà, si donchs lo posseydor fer no volrà compliment de dret. E si per aventura negú los laurarà, après lo dit comoniment fet per III vegades e per aquela raó dan pendrà no sia demanat per pau trencada, salva emperò la pau de les bèsties dades en lo hus de laurar, e d'aquels qui aqueles governaran o guardaran, ab totes les cosses que ab els portaran, car no volem que per contumàcia de pageses, les bèsties areges sien robades, esvaydes ho perdudes.

[XVI]. Les careres públiches, o camins, o estrades, en aital seguretat posam e establim, que negú los caminans o aquen pasans no esvaesca, ne en cors propi, ne a les lurs cosses, en neguna cosa iniúria ne greuge no faça, si donchs no seran cavalers o homens de gerra, pena lese mayestat aparexen a aquel qui contra assó farà, après satisfació en doble de les malefetes, e satisfet encara de la iniúria e del dan donat a aquel qui sofert ho aurà. 
[XVII]. Sots aquesta mateixa pau establim cavalers e tots aquels qui iran ab dona muler de cavaler, si donchs no seran baadors o trädors manifests, los quals d'aquesta pau e treves de tot en tot gitam./fol. 36 r.

[XVIII]. Los traïdors de lurs senyors, qui segons la constitució escrita la lur innocència purgar no volran, d'aquesta pau e seguretat axí els com los lurs honors, com encara los parcers o consintens ho ajudadors d'aquels, fors gitam e exceptats.

[XIX]. Les salvetats encara de tota la terra nostra, axí noves com antigament establides, sots la damunt dita pau e seguretat posam e establim.

[XX]. Fures o ladres, de nuit o de dia, e los lurs acuylidors, si esmenar lo mal que fet auran, no volran, e encara dret fer menysprean, de la damunt dita pau e seguretat aquels gitam, ab totes les lurs cosses, mobles e no mobles.

[XXI]. Si alcú, emperò, cavaler o ciutadà, contra aquesta manera de constitució vendrà, lo dan esmen simple a aquel al qual lo malfet aurà dins $\mathrm{XV}$ dies après que amonestat serà. $\mathrm{E}$ après los $\mathrm{XV}$ dies passats, en doble los esmen, e encara do CXX sous de moneda de doblenc, e payès emperò XL sous al bisbe e a nos, a qui los clams de pau e treves trencades seran pervenguts. $\mathrm{E}$ si dins los XV dies primers vinens, lo trencador de la establida pau e treves no esmenarà simple, so és saber per I vegada sens altra pena après, axí que la meitat d'aquest doble aya lo clamant, e l'altra meytat lo bisbe, e nos, qui aquesta justícia faedora aurem aquel sostengut. E encara si dins los damunt dits XV dies, per nos o per lo bisbe, o per missatge o missatges nostres, aquel trencador damunt dit, lo dit dan no esmenarà, d'aqui enant aquel malfetor e los parcers/fol. $36 u$ e ajudadors e conseyladors d'aquel, per lo bisbe sien vedars, e de la damunt dita pau e treves atrosí sien enteses, foragitars ab totes les lurs cosses, axí que mal que fer açó a els sia fet, no sia demanat per pau ne treva trencada. Mas si lo malferor e los ajudadors d'aquel al damunt dit clamant nuyl mal faran, sia esmenat per pau trencada.

[XXII]. Esters és d'establir, e de observar fermament per aquestes cosses, sots aquela pau e treves jutgam, los diomenges e les festes de tots los sants apòstols, e dels evangelistes, e l'Aveniment de Nostre Senyor Thesu Christ, entró a les vuitades d'Aparici, e la Quaresma, entró a les vuitades de Cinquagèsima, e lo dia encara de l'Ascensió, e lo dia de Cinquagèsima ab les sues huytades, e IIII festes de Nostra Dona Sancta Maria, e la festa de sant Iohan Baptista, e de sent Michel, e de Omnia Sentor, e les festes de senta Eulalia de Barchinona, e de sant Feliu de Gerona, e de sent Martí.

[XXIII]. Los trencadors encara, de pau, sien tenguts de fermar e de penyores tinents metre en mà del veger nostre, en pròpies persones. Si emperò, los senyor qui aquels al malefici aurà menars, $\mathrm{o}$ la gerra volrà, fer- 
mar e metre penyores per si e per los cavalers, e per los homens qui de casa e de companya sua seran, sia açó reebut.

[XXIV]. Si emperò, homens de monestirs o d'altres lochs religioses, entre els dan se daran, en cors ho en cosses, e los clams/fol. $37 \mathrm{r}$. aquests al veger vendran, trameta aquel o aquels als lurs propis senyors. E si dins XV dies aquel dan en poder de lurs senyors, adobar no volran, d'aquí avant lo veger nostre prena penyores tinents en persones pròpies, e aquel plet per fi deguda sia determenat.

[XXV]. Encara establim e manam que aquestes paus e treves sien fermes, e per tots temps duradores.

[XXVI]. Encara establim c manam que tots los cavalers e ciutadans, $\mathrm{e}$ homens de viles, de XIIII ayns $e$ de plus, aquestes paus iuren que les tengen e les defenen feelment, sens frau e tot engan. E qui après amonestació del bisbe o del veger nostre iurar no les volrà, la donchs sia vedat, e de pau e de treves gitat.

A maior encara seguretat, nos, en lacme, per la gràcia de Déu rey d'Aragó, comte de Barchinona e senyor de Monpesler, totes les cosses damunt dites e sengles iuram per Déu e per los sants IIII Evengelis, tenir fermament observar e fer observar.

Datum Barchinone, XII kalendas ianuarii, anno Domini millesimo CCo XXo VIII.

Sig +num lacobi, Dei gracia regis Aragonum et comitis [Barchinone] 16

Sig +num d'en Nuno Sanç, comte de Rosseyó e de Cerdanya. Sig+num d'en Hugo, comte d'Ampúries, qui açó iur, salva la del comdat d'Ampúries e de Perelada. Sig+num G. de Munchada, vescomte/fol. $38 v .^{17}$ de Biarn. Sig +num R. de Munchada, e d'en G. de Cervera, e d'en Hug de Mataplana, e d'en Bernat Hug de Seralonga, e d'en G. de Sent Vicenç, e d'en Gauçeran de Pinós, e d'en G. de Cervelyó, e d'en R. Alamayn, e d'en G. de Clarmunt, e d'en G. de Terraça, e d'en Dalmau, veçcomte de Rochabertí, e d'en Bernat de Sancta Eugènia, e d'en P. Berenguer de Riudeperes, e d'en Bernat de Baç, e d'en R. Berenguer d'Àger, e d'en R. de Gironela, e d'en Bernat de Sent Vicenç, d'en G. de Banyeres, e d'en Ponç da Oltrera, e d'en A. de Vídues, e d'en A. d'Uyldemolins, e d'en A. de Valvert. Nos, tots damunt dits, e sengles totes les damunt escrites cosses e sengles, iuram per Déu e per los sants IIII Evangelis, complir e servar, e fer servar axí com damunt és contengut.

Sig+num d'en G., escrivà, qui de manament del senyor rey, e per G. Rabasça, notari seu, assó escriure fou loc e dia e ayn damunt dit.

17. El foli 37 v. és en blanc. Al foli 38 r. es comencen a copiar les actes de la Cort General de Tarragona de 1235, en la seva versió llatina. 